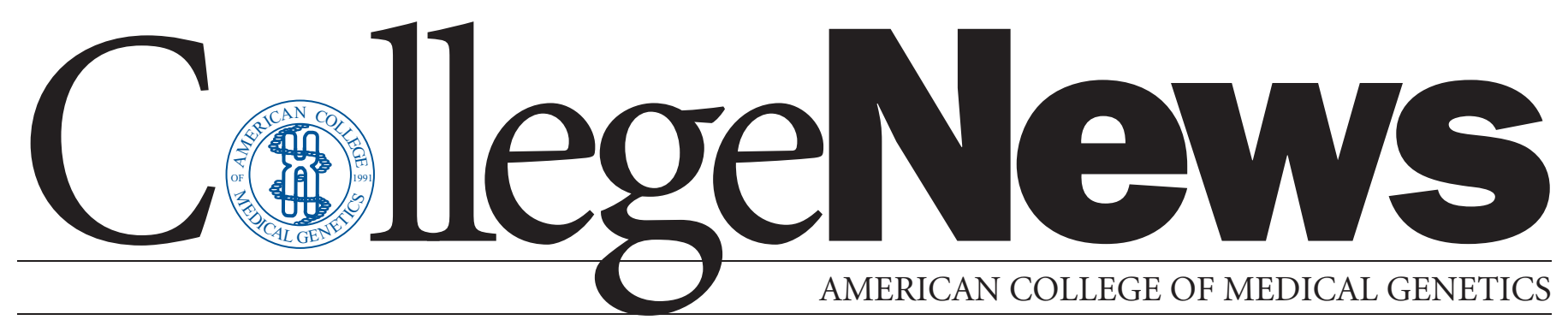

\section{Position Announcement}

\section{Director of Cytogenetics}

\section{Department of Medical Genetics, Henry Ford Health System, Detroit, Michigan}

The Henry Ford Department of Medical Genetics has an active and very well-integrated clinical genetics service, including local and outreach genetics clinics, and DNA Diagnostic, Cytogenetics, and Maternal Serum Screening Laboratories. We support the Henry Ford Health System (HFHS is the largest integrated medical system in Michigan; http://www.henryfordhealth.org) and many other hospitals and clinics throughout southeastern Michigan. The Henry Ford Medical System is strong and growing. The System will build a new 350-bed hospital in West Bloomfield, Michigan. We are presently seeking an enthusiastic and experienced board-certified clinical cytogeneticist.
The primary responsibility of this individual will be direction of the Cytogenetics Laboratory and communication with referring physicians. The laboratory volume will be approximately 6000 karyotype and 1200 FISH studies in 2003. The laboratory provides services to the Henry Ford Medical Group physicians, the Henry Ford Health System hospitals, and is a major reference laboratory for other hospital systems.

There are many teaching and research opportunities. Intramural grant programs are available to develop preliminary results for external (e.g., NIH) grant applications, and we have a well-regarded ABMG/RRC accredited training program in Medical Genetics.

Faculty appointments and compensation are commensurate with qualifications and experience. HFMG is an equal opportunity employer.

Letters of interest should be addressed to Kristin Monaghan, PhD, Department of Medical Genetics, Henry Ford Medical Group, 2799 West Grand Blvd., Detroit, MI 48202. 